\title{
Multi-class resource sharing with batch arrivals and complete blocking
}

\author{
Paul Ezhilchelvan and Isi Mitrani \\ School of Computing Science, Newcastle University, NE1 7RU, UK \\ e-mail: paul.ezhilchelvan@ncl.ac.uk, isi.mitrani@ncl.ac.uk
}

\begin{abstract}
A cloud provider hosts virtual machines of different types, with different resource requirements. There are bounds on the total amounts of each kind of resource that are available. Requests arrive in batches of different sizes, and are accepted if all the VMs in the batch can be accommodated; otherwise the request is blocked, with an associated loss of revenue. The trade-offs between costs and benefits are evaluated by means of an appropriate model, for which a novel solution is proposed. The applicability of that solution is extended, by means of a simplification, to very large-scale systems. Numerical examples and comparisons with simulations are presented.
\end{abstract}

\section{Introduction}

A cloud provider may offer services of different types, with different patterns of demand, resource requirements and charges. A job of a given type is run by instantiating an appropriate Virtual Machine (VM), provided that the resources it requires are available. There are bounds on the total amounts of different resources, so that whether a VM can be instantiated or not, depends both on the type of the new job and on the numbers and types of the other jobs already running.

We are concerned with systems where user demands arrive in batches whose sizes may be fixed or random, and may depend on type. Moreover, the nature of the applications is such that users are not interested in partial acceptance: either all VMs in a batch must be instantiated, or none. This is known as the complete blocking policy, in contrast to the partial blocking policy whereby a part of a batch may be accepted and the rest rejected.

There are many applications which require a batch of VMs in order to complete a certain task within a certain period of time. These are often concerned with the analysis of large volumes of data, such as those arising in the fields of sociology, biology or high energy physics. In particular, the 'MapReduce' framework (e.g., see [3]), allowing the deployment of batches of VMs, is widely used.

The trade-offs in this context are between the costs incurred by providing resources, and the revenues obtained by running jobs. The problem is to decide what amounts of the various resources to provide, so as to maximize the average long-term profit (revenues minus costs) per unit time, or to achieve certain 
quality-of-service objectives. To that end, we analyze and solve an Erlang-type loss model with multi-class batch arrivals and complete blocking.

We assume that the demand parameters are given, and the system reaches steady state during a period where those parameters remain fixed. In practice, the resource provisioning policies would have to be supplemented by some monitoring and parameter estimation technique that would detect when the traffic parameters change. Such techniques exist (see below). It is also worth pointing out that batch arrivals can, and have been, used to model bursty arrival streams.

The model that is addressed here has been considered before, but has not been solved. In their 1995 paper [2], Choudhury, Cheung and Whitt claimed to provide a product-form solution for both the partial blocking and the complete blocking policies. That solution agreed with the results obtained by Kaufman and Regge [9], for general distribution of batch sizes, and by van Doorn and Planken [4], for geometrically distributed batches. However, both of those papers had analyzed only the partial blocking case.

We agree that a product-form solution holds in systems with partial blocking of batches, but will show that it does not hold in the case of complete blocking. Therefore, that problem is still open. The purpose of the present paper is to fill some of the gap by proposing and evaluating an accurate approximate solution based on fixed-point iterations.

The rest of the existing literature on multi-class resource sharing deals mainly with demands arriving one at a time in Poisson streams (i.e., no batches). Much of the work is in the context of circuit-switched networks, e.g., Kelly [10], Hampshire et al [7], Kaufman [8], Roberts [14] and Ross [15]. In the telephony field, the resources are the circuits available on various links, and the job types are indexed by the set of links that can be reserved for a call. The optimal allocation of VMs on servers hired from a cloud was explored in Ezhilchelvan and Mitrani [6], and in Tan et al [17]. Again, one-at-a-time Poisson arrivals were assumed.

More distantly related is quite a large body of work on server allocation with a single job type. Ezhilchelvan and Mitrani [5] showed that dynamic allocation policies do not bring significant benefits over static ones. The trade-off between performance and energy consumption was examined by Mazzucco et al. [11, 12], using models and empirical observations. Their focus, and also that of Bodík et al. [1], was on estimating the traffic and reacting to changes in the parameters.

The model, and the profit maximization problem, are described in section 2 . The solution presented by Choudhury et al is shown to be erroneous in section 3 , while section 4 describes and evaluates the proposed fixed-point approximation. Section 5 is concerned with very large-scale systems. A simplified version of the fixed-point approximation is shown to be accurate and numerically stable. Some conclusions and directions for future work are summarized in section 6 .

\section{The model}

The hosting infrastructure provides $R$ different types of resources, such as CPUs, memory, interconnection bandwidth, etc. The total amount of available resource 
of type $r$ is $D_{r}$, referred to as the 'resource capacity' of type $r(r=1,2, \ldots, R)$. Those resources are shared by VMs, or jobs, of $T$ different types. A job of type $j$ requires an amount $d_{j, r}$ of type $r$ resource $(j=1,2, \ldots, T ; r=1,2, \ldots, R)$. In order to run a job, all its resource requirements must be satisfied. For every $j$, at least one of the requirements $d_{j, r}$ is greater than 0 , which imposes a limit on the maximum number, $m_{j}$, of type $j$ jobs that can run in parallel:

$$
m_{j}=\min _{r}\left\{\left\lfloor\frac{D_{r}}{d_{j, r}}\right\rfloor\right\}
$$

where $\lfloor x\rfloor$ is the largest integer less than or equal to $x$.

Moreover, if $D_{r}$ is replaced by the type $r$ resource capacity currently available (determined by the numbers and types of jobs currently running), then (1) provides a limit on the number of type $j$ jobs that can be admitted at a given moment.

Requests of type $j$ arrive in an independent Poisson stream with rate $\lambda_{j}$. Each such request consists of a batch of jobs, all of type $j$, whose size has an arbitrary distribution dependant on $j$ : there are $k$ jobs in the batch with probability $q_{j, k}$. If there is at least one job in the batch that cannot be run because at least one of the resources it requires cannot be provided, then the whole batch is rejected. That is the complete blocking policy.

The probabilities $q_{j, k}$ can be arbitrary. However, a sensible batch size distribution should be consistent with (1). In other words, there should be a limit, $K_{j}$, on batch sizes of type $j$, such that $K_{j} \leq m_{j}$. Otherwise, some batches would be rejected even if there are no other jobs present.

Service times for jobs of type $j$ are assumed to be i.i.d. random variables distributed exponentially with mean $1 / \mu_{j}(j=1,2, \ldots, T)$. The insensitivity property of the Erlang model does not hold when jobs arrive in batches (see [8]).

Suppose that each unit of resource of type $r \operatorname{costs} c_{r}$ to provide, and each job of type $j$ that is run brings in a revenue of $v_{j}$. Denote by $\alpha_{j, k}$ the steady-state probability that an incoming batch of type $j$ and size $k$ is accepted. Then the total steady-state average profit, $V$, that the system generates per unit time, can be expressed as

$$
V=\sum_{j=1}^{T} \lambda_{j} \sum_{k=1}^{K_{j}} q_{j, k} \alpha_{j, k} k v_{j}-\sum_{r=1}^{R} c_{r} D_{r}
$$

Clearly, increasing the resource capacities $D_{r}$ leads to higher revenues, but also higher costs. The profit optimization problem is to choose $D_{r}$ so as to maximize $V$. One could also consider a Quality-of-Service problem, which is to find the minimum values of $D_{r}$ for which the acceptance probabilities $\alpha_{j, k}$ exceed certain pre-defined targets. In both cases, it is necessary to solve the model in order to determine $\alpha_{j, k}$. 


\section{An erroneous solution}

The technique used in Choudhury et al [2] is to replace an incoming batch of type $j$ and size $k$ (when $q_{j, k}>0$ ), by a single macro job that goes through a series of $k$ queues in tandem: in the first queue it uses $k d_{j, r}$ units of type $r$ resource and is served at rate $k \mu_{j}$, regardless of how many other such macro jobs are present; in the second queue it uses $(k-1) d_{j, r}$ units of resource and is served at rate $(k-1) \mu_{j}$; this goes on until queue $k$, where it uses $d_{j, r}$ units of resource and is served at rate $\mu_{j}$. After that, the macro job departs.

In this formulation, which is equivalent to the one in terms of batches of ordinary jobs, the system state is a vector of integers $\left[n_{j, k, s}\right]$, specifying the numbers of macro jobs of type $j$ and size $k$ that are now in queue $s$ of their series of queues (i.e., they have $k+1-s$ ordinary jobs remaining). The authors find that, for both partial and complete blocking, the probabilities of those vectors satisfy local balance, and therefore the steady-state distribution has product form:

$$
\pi(\mathbf{n})=G \prod_{j=1}^{T} \prod_{k=1}^{K_{j}} \prod_{s=1}^{k} \frac{\left(\lambda_{j} q_{j, k}\right)^{n_{j, k, s}}}{\left((k+1-s) \mu_{j}\right)^{n_{j, k, s}} n_{j, k, s} !},
$$

where $\mathbf{n}$ is the state vector $\left[n_{j, k, s}\right]$ and $G$ is a normalization constant.

To demonstrate that this product form does not hold in the case of complete blocking, we offer the following simple counter-example. Take a system with a single resource type, a single job type, and a single batch size. The resource capacity is 4 , the resource requirement per job is 1 and the incoming batch size is 3 . The arrival and service rates are both 1 .

There are now 3 queues in series, so the system state is a triple $\left(n_{1}, n_{2}, n_{3}\right)$. The feasible states are $(0,0,0),(1,0,0),(0,1,0),(0,0,1),(1,0,1),(0,1,1)$ and $(0,0,2)$. The state $(0,2,0)$, for instance, is not feasible because if there was a macro job in queue 2, consuming 2 resource units, then a new macro job requiring 3 units would not have been admitted.

Consider the two states $(0,0,1)$ and $(1,0,1)$. If $(3)$ is correct, then their stationary probabilities are $\pi(0,0,1)=G$ and $\pi(1,0,1)=G / 3$. Hence, $\pi(0,0,1)=$ $3 \pi(1,0,1)$. On the other hand, the only way of leaving state $(1,0,1)$ is by a service completion, either at queue 1 , at rate 3 , or at queue 3 , at rate 1 . The total completion rate is 4 . The only way of entering state $(1,0,1)$ is by an arrival of a new batch, at rate 1 , when the system is in state $(0,0,1)$. Therefore, $\pi(0,0,1)=4 \pi(1,0,1)$. This contradiction demonstrates that the solution (3) is not correct.

The failure of the product form is due to the fact that, contrary to the assertion in [2], local balance does not hold in the case of complete blocking. In the above example, in states $(1,0,1)$ and $(0,1,1)$, a service completion at queue 3 (leading to states $(1,0,0)$ and $(0,1,0)$ respectively), cannot be balanced by an arrival because in either case the new batch would be rejected. For that reason, although in principle there might exist a different, as yet undiscovered product form, we believe that this is very unlikely. 
In the absence of a tractable exact solution, we now turn to the task of finding an accurate approximation.

\section{A fixed-point approximation}

We propose treating each job type as if it was an isolated, one-dimensional Markov process taking place within a static environment determined by the other job types. More precisely, when considering jobs of type $j$, assume that all jobs of other types in the system are consuming a fixed total amount, $Z_{j, r}$, of type $r$ resource $(r=1,2, \ldots, R)$. In other words, type $j$ operates in an environment where the available resource of type $r$ is $D_{r}-Z_{j, r}$. Hence, the maximum number of type $j$ jobs that can be admitted, $m_{j}$, is given by (1), with $D_{r}$ replaced by $D_{r}-Z_{j, r}$.

Under the above assumption, the type $j$ Markov process evolves on the state space $\left\{0,1, \ldots, m_{j}\right\}$. Let $\pi_{j}(n)$ be the stationary probability of state $n$, i.e., the probability that there are $n$ type $j$ jobs present. We can write a set of recurrence relations for these probabilities by examining the flows across a cut separating states $0,1, \ldots, n-1$ from states $n, n+1, \ldots, m_{j}$. The relevant balance diagram is illustrated in Figure 1.

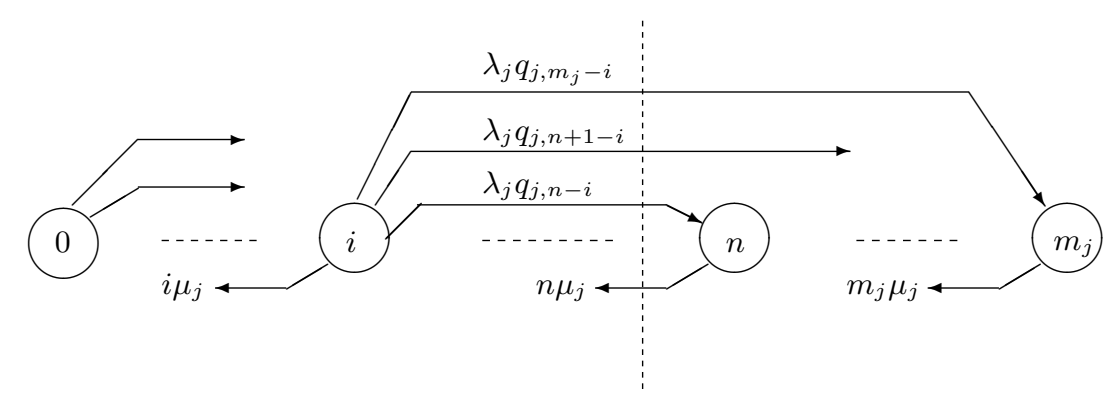

Fig. 1. Balance diagram for type $j$

The downward (i.e., to the left) flow across the cut contains just a single transition, from state $n$, due to a departure; the corresponding rate is $n \mu_{j}$. On the other hand, if an arrival occurs in any state, $i(i<n)$, and the size of the incoming batch is at least $n-i$ but does not exceed $m_{j}-i$, then it contributes to the upward (to the right) flow across the cut. Equating the two flows provides a balance equation for each $n$ :

$$
n \mu_{j} \pi_{j}(n)=\lambda_{j} \sum_{i=0}^{n-1} \pi_{j}(i) \sum_{k=n-i}^{m_{j}-i} q_{j, k} ; n=1,2, \ldots, m_{j}
$$


The simplest way to solve these equations is to set $\pi_{j}(0)=1$, evaluate $\pi_{j}(n)$ for $n=1,2, \ldots, m_{j}$ according to (4), and then re-normalize by dividing each of them by their sum.

Having computed the probabilities $\pi_{j}(n)$, the probability that an incoming batch of type $j$ and size $k$ is accepted, is given by

$$
\alpha_{j, k}=\sum_{n=0}^{m_{j}-k} \pi_{j}(n) .
$$

The average number, $L_{j}$, of type $j$ jobs present is equal to

$$
L_{j}=\sum_{n=1}^{m_{j}} n \pi_{j}(n) .
$$

Consequently, the average amount, $z_{j, r}$, of type $r$ resource consumed by jobs of type $j$, is given by

$$
z_{j, r}=d_{j, r} L_{j}
$$

In common with most models of this type, the above solution depends only on the ratio $\rho_{j}=\lambda_{j} / \mu_{j}$, and not on the individual values of those parameters.

We can now approximate the effect that type $j$ has on the other job types by treating its average consumption of resource $r, z_{j, r}$ as a fixed consumption of resource $r$. This will form part of the environment in which another given job type operates.

Suppose that we have somehow obtained an estimated vector, $\mathbf{L}=\left(L_{1}, L_{2}\right.$, $\left.\ldots, L_{T}\right)$, of the average numbers of jobs of different types in the system. Carry out the following procedure.

1. For every $j$, compute the type $r$ resource, $Z_{j, r}$, consumed by job types other than $j$ :

$$
Z_{j, r}=\sum_{i=1, i \neq j}^{T} z_{i, r}
$$

with $z_{i, r}$ being given by (7).

2. For every $j$, solve the isolated Markov process of type $j$ and compute a new value for $L_{j}$ (and hence new values for $z_{j, r}$ and the acceptance probabilities $\left.\alpha_{j, k}\right)$.

This procedure implements a mapping, $f(\cdot)$, from one vector of averages, call it $\mathbf{L}^{\text {old }}$, to another vector of averages, $\mathbf{L}^{\text {new }}$. Our approximate solution consists in finding a vector, $\mathbf{L}^{*}$, whose 'new' image is the same as the 'old' one. That is, $\mathbf{L}^{*}$ is a fixed point of the mapping $f(\cdot)$ :

$$
\mathbf{L}^{*}=f\left(\mathbf{L}^{*}\right) .
$$

At the fixed point $\mathbf{L}^{*}$, the environments in which the different job types operate are consistent with each other. That is, for every job type $j$, the resources 
it consumes, $z_{j, r}$, do not alter the resources consumed by the other job types, $Z_{j, r}$. In that sense, this fixed point is like a Nash equilibrium in multi-person games.

The acceptance probabilities corresponding to $\mathbf{L}^{*}$ are substituted into (2) in order to compute the profit $V$, or are used to see whether the QoS targets have been met.

To compute $\mathbf{L}^{*}$, we use an iterative schema of the form

$$
\mathbf{L}^{(i+1)}=f\left(\mathbf{L}^{(i)}\right) .
$$

These iterations start with some initial vector such as $\mathbf{L}^{(0)}=(0,0, \ldots, 0)$, and stop when two consecutive vectors are sufficiently close to each other. To reduce the number of iterations, it is advisable that the evaluations are of the Gauss-Seidel type, i.e. as soon as a new value for some $L_{j}$ is obtained, the corresponding value of $z_{j, r}$ is used in determining the environment for other job types.

Note. Fixed-point approximations of queuing systems have been used in the past, mainly in the context of open or closed networks. There, the decomposition is in terms of nodes and the fixed point equations attempt to capture the interactions between them (e.g., see Sadre et al. [16], Whitt [18]). Kelly's fixedpoint approximation for multi-class circuit-switched networks [10] is concerned with shared resources, but does not model batch arrivals. The decomposition is with respect to offered loads for individual units of resource. Kelly was able to prove the existence of a fixed point by appealing to Brouwer's theorem, and he demonstrated convergence to it.

We have been unable to prove either the existence or the uniqueness of a fixed point for our mapping $f(\cdot)$. This is due to the fact that equation (1) involves the 'floor' function $\lfloor x\rfloor$, which is not continuous. However, $f(\cdot)$ is uniformly bounded and our experience with many examples has been that the iterations (10) always converge.

As far as we are aware, a decomposition by job type, where the fixed point equations capture different contributions to a shared environment, has not been used before.

To examine the quality of the proposed approximation, consider an example system with four job types, 1, 2, 3 and 4, or 'small', 'medium', 'large' and 'very large'. There is a single resource type and the individual resource requirements of the four job types are are $d_{1}=1, d_{2}=2, d_{3}=4$ and $d_{4}=8$. These numbers are motivated by similarities with the T2 family of VM instances offered by the Amazon EC2 (Elastic Computing Cloud) service (see [19]). The resource that is being shared in this context is vCPUs (virtual CPUs).

Type 1 jobs arrive singly, at a rate of $\lambda_{1}=6$ jobs per unit time. Their average service times are $1 / \mu_{1}=1$. For type 2 , the possible batch sizes are 1 or 2 , with equal probability $\left(q_{2,1}=q_{2,2}=0.5\right)$. The traffic parameters are $\lambda_{2}=4$ and $1 / \mu_{2}=1$. Jobs of type 3 and type 4 arrive in batches of size 1,2 , or 3 , with probabilities $0.4,0.3$ and 0.3 , respectively. Their arrival and service parameters are $\lambda_{3}=2,1 / \mu_{3}=0.5, \lambda_{4}=1,1 / \mu_{4}=0.5$. 
The cost incurred per unit of resource is $c=0.2$, and the revenues brought in by the different job types increase with the resource consumed: $v_{1}=1, v_{2}=3$, $v_{3}=6, v_{4}=10$.

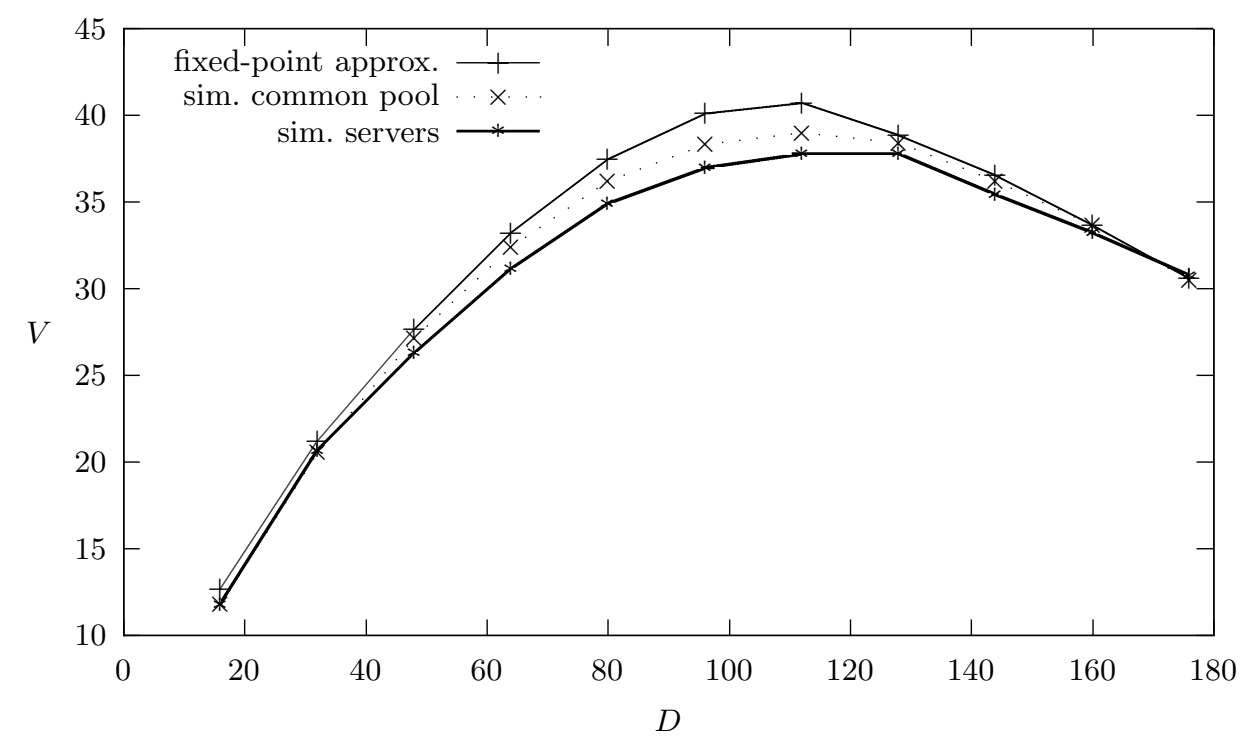

Fig. 2. Estimated and simulated average profit

In Figure 2, the estimated average profit is plotted against the offered resource capacity, $D$. The three curves correspond to (a) the fixed-point approximation, (b) a simulation of the model as described in section 2 and (c) a simulation of a system where the resource is not provided as a common pool, but is contained within servers. In this last case, each server has 16 vCPUs. Thus, in order to offer 128 vCPUs, one has to provide 8 servers. The discrete server system is less efficient than the common pool, because, in order to instantiate a VM on a server, all its resource requirement must be available on that server. It is not enough to have some of the requirement available on one server and some on another.

The two simulation graphs may be considered exact. Their confidence intervals are too narrow to plot. The figure shows that the fixed-point approximation tends to overestimate the average profit slightly. This is not surprising, since replacing a random environment with a fixed one reduces the variance of the process. The relative differences between approximated and simulated (common pool) points are on the order of $5 \%$ or less.

The discrete server simulation confirms the intuition that that system is less efficient, but again the differences from the common pool are on the order of $5 \%$ 
or less. In particular, all three graphs agree that the optimal resource capacity to provide is $112 \mathrm{vCPUs}$, or 7 servers.

The convergence of iterations (10) to the fixed point is very fast. The termination criterion in this example was that $\left|L_{j}^{(i+1)}-L_{j}^{(i)}\right|<10^{-6}$ for all job types $j$. The entire approximation graph took less than tenth of a second to compute, and no point required more than 4 iterations.

We have also simulated a discrete server system where each server contains 8 vCPUs instead of 16 . The differences between that system and and the one shown in the figure are negligible.

In the next example, all arrival rates are scaled up by a factor of 100: $\left(\lambda_{1}, \lambda_{2}, \lambda_{3}, \lambda_{4}\right)=(600,400,200,100)$. The resource is again provided in a common pool, with scaled capacities ranging from 7000 to 10000 . The other parameters, revenues and costs are kept as before. The aim of this exercise is to see whether the fixed-point approximation is sufficiently robust to cope with the much larger state space and offered loads. The estimated and simulated average profits are displayed in Figure 3.

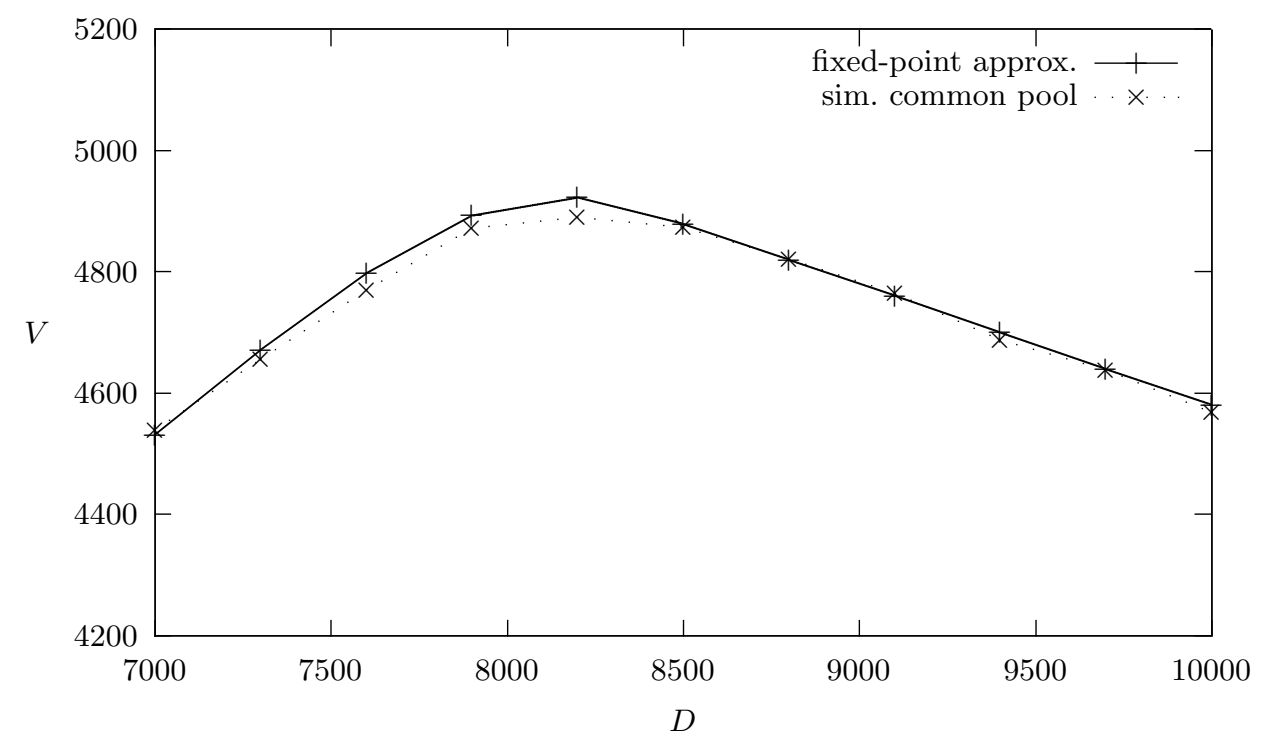

Fig. 3. System scaled up by a factor of 100

The accuracy of the approximation has increased. Not only does the model predict the optimal resource allocation correctly, but the relative differences between estimated and simulated profits are now less than $1 \%$. This is not really surprising, since the flows of traffic of various job types through the system 
behave more and more like deterministic fluids when capacities and offered loads increase.

We conclude that, for systems of this and similar sizes, it would be preferable to use the fixed-point approximation even if an exact product-form solution was available. The reason is that the complexity and numerical problems associated with computing the relevant normalization constants increase very quickly with the number of job types, the incoming batch sizes and the offered resource capacities.

It should be acknowledged, however, that the fixed-point approximation also has its limits of applicability. For example, if the system in Figure 3 is scaled up by another factor of 10 , bringing the arrival rates to $\left(\lambda_{1}, \lambda_{2}, \lambda_{3}, \lambda_{4}\right)=$ $(6000,4000,2000,1000)$ and resource capacities in the region of 100000 , our solution breaks down. The failure is not in the fixed-point iterations, but in the one-dimensional solutions (4) which start experiencing numerical overflows.

It is thus desirable to develop another approximation which can be applied to very large systems and produce reasonable estimates, albeit with some loss of accuracy.

\section{$5 \quad$ Very large-scale systems}

We have observed that the solution of an isolated job type ceases to work when the resource capacities and the offered loads are on the order of tens of thousands or more. For such large systems, a simpler and more robust approximation is required.

With this in mind, we propose to represent the various batch arrivals of type $j$ by single 'macro' jobs with appropriately chosen resource requirements. Then, for the purpose of the fixed-point solution, the isolated type $j$ model becomes a classic Erlang loss process. The benefit of this simplification is that the Erlang B function, which provides the rejection probability, can be computed in a stable manner for large values of the parameters.

The arrival rate and average service time of type $j$ macro jobs are $\lambda_{j}$ and $1 / \mu_{j}$, respectively. To define the resource requirement of type $r$ for a macro job of type $j, \delta_{j, r}$, we take the average over the possible type $j$ batch sizes:

$$
\delta_{j, r}=d_{j, r} \sum_{k=1}^{K_{j}} k q_{j, k} .
$$

Hence, if all other job types consume a fixed amount, $Z_{j, r}$, of type $r$ resource, then the maximum number of type $j$ macro jobs that can be admitted into the system is

$$
m_{j}=\min _{r}\left\{\left\lfloor\frac{D_{r}-Z_{j, r}}{\delta_{j, r}}\right\rfloor\right\} .
$$


The probability, $\beta_{j}$, that an incoming macro job of type $j$ will be rejected, is given by the Erlang-B function (eg, see [13])

$$
\beta_{j}=B\left(m_{j}, \rho_{j}\right)=\frac{\rho_{j}^{m_{j}}}{m_{j} !}\left[\sum_{i=0}^{m_{j}} \frac{\rho^{i}}{i !}\right]^{-1} .
$$

A numerically stable procedure for computing the function $B(m, \rho)$ is provided by the recurrence relation

$$
B(m, \rho)=\frac{\frac{\rho}{m} B(m-1, \rho)}{1+\frac{\rho}{m} B(m-1, \rho)},
$$

starting with $B(0, \rho)=1$ (e.g., see $[6]$ ).

The average number, $L_{j}$, of type $j$ macro jobs in the system is then given by Little's result:

$$
L_{j}=\rho_{j}\left(1-\beta_{j}\right) .
$$

The average amount of type $r$ resource that those jobs consume is $z_{j, r}=L_{j} \delta_{j, r}$.

We now have the necessary elements for carrying out the iterations described in the previous section and finding the fixed-point vectors of average numbers of macro jobs present, $L_{j}^{*}$, and corresponding rejection probabilities, $\beta_{j}^{*}$. The average profit achieved per unit time is given by

$$
V=\sum_{j=1}^{T} \lambda_{j} v_{j}\left(1-\beta_{j}\right) \sum_{k=1}^{K_{j}} k q_{j, k}-\sum_{r=1}^{R} c_{r} D_{r} .
$$

To illustrate the efficacy and accuracy of this approximation, we have scaled up the example with four job types introduced in the previous section. The arrival rates are now $\left(\lambda_{1}, \lambda_{2}, \lambda_{3}, \lambda_{4}\right)=(6000,4000,2000,1000)$, and the single resource capacity is varied in the range $D \in(70000,90000)$. All other parameters are as before.

In Figure 4, the average profits predicted by the macro fixed-point approximation are compared with those produced by simulation runs in each of which a total of ten million batches of all types and sizes arrived into the system. Computing the fixed-point was very fast and free from numerical problems. The simulation runs were several orders of magnitude slower, because we wanted narrow confidence intervals.

The figure shows that the simplified approximation is remarkably accurate at this scale. The two plots are almost indistinguishable. This confirms the tendency observed earlier, that as the scale of the system increases, its behaviour agrees more closely with the deterministic assumptions that underlie the fixed-point approach.

\section{Conclusions}

We have addressed a practically relevant problem concerned with service provisioning in public clouds. The multi-class model with batch arrivals and complete 


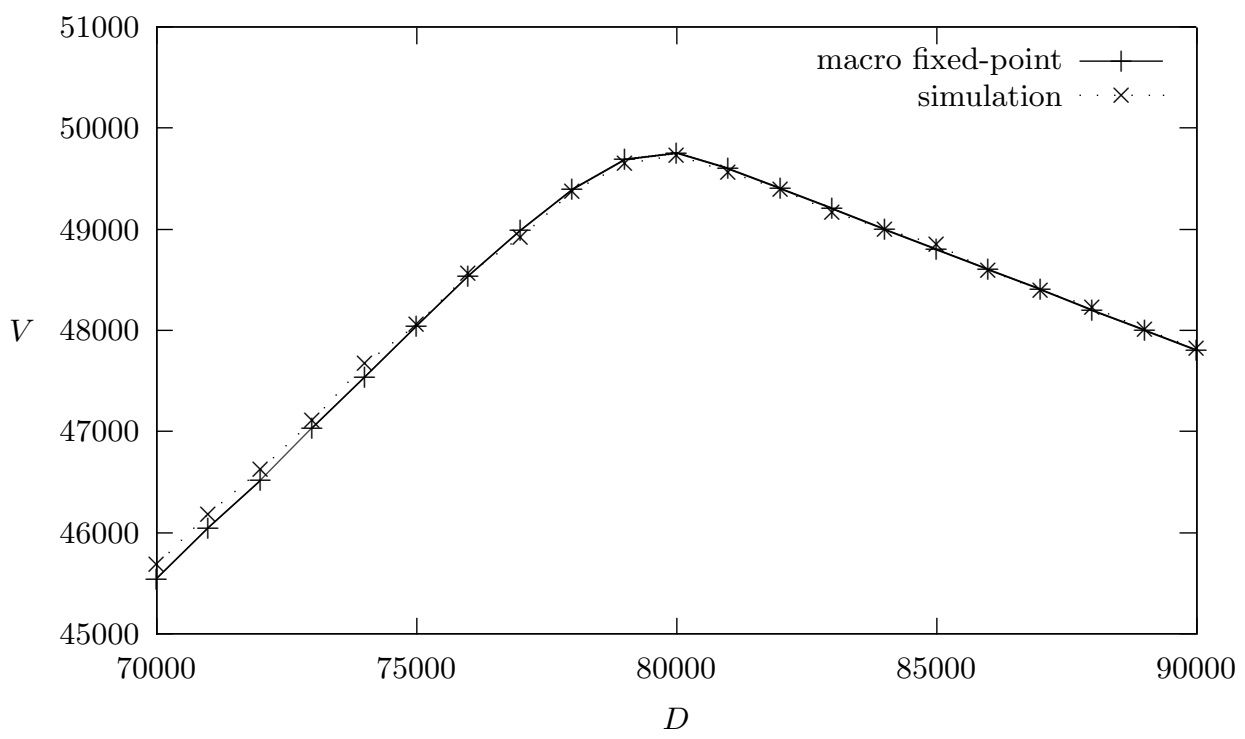

Fig. 4. System scaled up by a factor of 1000

blocking appeared to be solved, but we have shown that the existing solution is incorrect. An alternative solution based on fixed-point iterations is introduced. This replaces the multi-dimensional stochastic process with a number of singledimensional ones, using averages to model the interactions between them. The accuracy of the fixed-point solution is good for small systems, and gets better when the system size increases. A simplified version of that solution is shown to apply to very large-scale systems.

The exact solution of the complete blocking model is still an open problem, as is also the solution of the model where resources are provided in discrete servers, rather than in common pools. Those problems are interesting and worthy of further study. However, we feel that even if the exact solutions were available, it would be better to tackle the task of optimizing a real-life system by using the proposed approximations. They are easily implementable and sufficiently accurate.

Other, more general models may be tackled by the methods described here. For example, one may wish to operate a complete blocking policy for some job types, and partial blocking for others. The one-dimensional solution for partial blocking is different, but the fixed-point approach would still apply.

\section{References}

1. P. Bodík, R. Griffith, C. Sutton, A. Fox, M. Jordan and D. Patterson, "Statistical machine learning makes automatic control practical for internet datacenters", Conf. 
on Hot Topics in Cloud Computing (HotCloud'09), Berkeley, CA, USA, 2009.

2. G.L. Choudhury, K.K. Leung and W. Whitt, "Resourse-sharing models with statedependent arrivals of batches", in Computations with Markov Chains (ed. W.J. Stewart), Kluwer, pp. 225-282, 1995.

3. J. Dean and S. Ghemawat, "MapReduce: Simplified data processing on large clusters", Communications of the ACM, 51, 1, pp. 107113, 2008.

4. E.A. van Doorn and F.J.M Planken, "Blocking probabilities in a loss system with arrivals in geometrically distributed batches and heterogeneous service requirements", ACM/IEEE Trans. on Networking, 1, pp. 664-667, 1993.

5. P. Ezhilchelvan and I. Mitrani, "Static and Dynamic Hosting of Cloud Servers", Computer Performance Engineering LNCS 9272, Eds. M. Beltran, W. Knottenbelt and J. Bradley), Springer, 2015.

6. P. Ezhilchelvan and I. Mitrani, "Optimal provisioning of servers for hosting services of multiple types", Simulation Modelling Practice and Theory, 75, pp. 17-28, 2017.

7. R.C. Hampshire, W.A. Massey, D. Mitra and Q. Wang, "Provisioning for Bandwidth Sharing and Exchange", in Telecommunications Network Design and Management, vol. 23 of series Operations Research/Computer Science Interfaces, Springer, pp. 207-225, 2003.

8. J.S. Kaufman, "Blocking in a shared resource environment", IEEE Trans. Commun., 29, pp. 1474-1481, 1981.

9. J.S. Kaufman and K.M. Rege, "Blocking in a shared resource environment with batched Poisson arrival processes", Performance Evaluation, 24, pp. 249-263, 1996.

10. F. Kelly, "Blocking probabilities in large cirquit switched networks", Advances in Applied Probability, 18, pp. 473-505, 1986.

11. M. Mazzucco, D. Dyachuk, and M. Dikaiakos, "Profit-aware server allocation for green internet services", IEEE Int. Symp. on Modeling, Analysis and Simulation of Computer and Telecommunication Systems (MASCOTS), pp. 277-284, 2010.

12. M. Mazzucco, M. Vasar, and M. Dumas, "Squeezing out the cloud via profitmaximizing resource allocation policies", IEEE Int. Symp. on Modeling, Analysis and Simulation of Computer and Telecommunication Systems (MASCOTS), pp. 19-28, 2012.

13. I. Mitrani, Probabilistic Modelling, Cambridge University Press, 1998.

14. J.W. Roberts, "A service system with heterogeneous user requirement", in Performance of Data Communications Systems and Their Applications, (Ed. G. Pujolle), North-Holland, pp. 423-431, 1981.

15. K.W. Ross, Multiservice Loss Models for Broadband Telecommunication Networks, Springer-Verlag, 1995.

16. R. Sadre, B.R. Haverkort and A. Ost. "An efficient and accurate decomposition method for open finite- and infinite-buffer queueing networks", Proc. 3rd Int. Workshop on Numerical Solution of Markov Chains (Eds. W. Stewart and B. Plateau), pp. 120, 1999.

17. Y. Tan, Y. Lu and C.H. Xia, "Provisioning for large scale loss network systems with applications in cloud computing", ACM SIGMETRICS Performance Evaluation Review, 40(3), pp. 83-85, 2012.

18. W. Whitt, "The Queueing Network Analyzer", Bell System Technical Journal, 62(9), pp. 27792815, 1983.

19. https://aws.amazon.com/ec2/instance-types/, Amazon Web Services, 2016. 